\title{
MINIMAL GENERATION OF FINITE SOLUBLE GROUPS BY PROJECTORS AND NORMALIZERS
}

\author{
A. LUCCHINI \\ Università degli Studi di Brescia, Via Branze 38, 25123 Brescia, Italy \\ and M. C. TAMBURINI \\ Università Cattolica del Sacro Cuore, Via Trieste 17, 25121 Brescia, Italy
}

(Received 19 September, 1997)

1. Introduction. In this paper $G$ denotes a non-identity finite soluble group. If $A$ is an irreducible $G$-module, $\operatorname{End}_{G} A$ is a division ring by Schur's Lemma, actually a field, since $G$ finite forces $A$ to be finite. Moreover $A$ is a vector space over $\operatorname{End}_{G} A$ with respect to $\alpha a:=\alpha(a), \alpha \in \operatorname{End}_{G} A, a \in A$. We let $\varphi_{G}(A):=\operatorname{dim}_{\operatorname{End}_{G} A} A$. Any chief factor of $G$ is an irreducible $G$-module via the conjugation action, and it is central precisely when it is a trivial $G$-module. By a refined version of the Theorem of Jordan-Hölder [1, p. 33] the number $\delta_{G}(A)$ of complemented chief factors of $G$, which are $G$-isomorphic to a given $A$, is constant for any chief series of $G$. We say that $A$ is complemented, as a G-module, if $\delta_{G}(A)>0$. Let

$$
\Omega(G):=\{\text { non-isomorphic, irreducible, complemented } G \text {-modules }\} .
$$

The following formula, for the minimal number $d(G)$ of generators of $G$, can be deduced from the work of Gaschütz [2]:

$$
d(G)=\max _{A \in \Omega(G)} h_{G}(A)
$$

where

$$
h_{G}(A):=\left[\frac{\delta_{G}(A)-1-\theta_{G}(A)}{\varphi_{G}(A)}\right]+2
$$

and $\theta_{G}(A):=1$ if $A$ is trivial, $\theta_{G}(A):=0$ otherwise.

For what follows our reference is [1]. Let $\mathfrak{X}$ be a Schunck class of characteristic $\pi$ in the universe $\subseteq$ of finite soluble groups. A $\pi$-group $G$ is generated by its $\mathfrak{X}$-projectors, which are all conjugate. We let $\eta_{\mathfrak{X}}(G)$ be the minimal number of $\mathfrak{X}$-projectors which generate $G$. In a similar way, if $\mathfrak{F}$ is a saturated formation in $\subseteq$ and the characteristic of $\mathfrak{\wp}$ is the set $\mathbb{P}$ of all primes, $G$ is generated by its $\mathfrak{\wp}$-normalizers. Again, they are all conjugate. We denote by $\tilde{\eta}_{\mathfrak{\Im}}(G)$ the minimal number of $\mathfrak{\wp}$-normalizers which generate $G$. The aim of this paper is to obtain formulas for the functions $\eta_{\mathfrak{X}}$ and $\tilde{\eta}_{\mathfrak{Y}}$ similar to the one of Gaschütz for the function $d$.

Let $H$ be an $\mathfrak{X}$-projector of $G$ and let $A \in \Omega(G)$. We show that, if $M_{1} / N_{1}$ and $M_{2} / N_{2}$ are complemented chief factors of $G$ that are $G$-isomorphic to $A$, then 
$M_{1} \cap H \leq N_{1}$ if and only if $M_{2} \cap H \leq N_{2}$. In this case we say that $H$ avoids $A$ and define

$$
\Omega_{\mathfrak{X}}(G):=\{A \in \Omega(G) \mid H \text { avoids } A\} .
$$

For a $\pi$-group $G$, we obtain the formula:

$$
\eta_{\mathfrak{X}}(G)=\max \left\{\max _{A \in \Omega_{\mathfrak{X}}(G)}\left\{h_{G}(A)\right\}, 1\right\} .
$$

In particular, when the Schunck class is a saturated formation $\mathfrak{\Im}, \Omega_{\mathfrak{\wp}}(G)$ actually consists of those $A$ 's in $\Omega(G)$ for which every $H$-chief factor of $A$ is $\widetilde{\lessgtr}$-eccentric.

Now assume, more generally, that $H$ is a subgroup of $G$ such that $H^{G}=G$. For each $\alpha \in \operatorname{End}_{G} A, \alpha\left(C_{A}(H)\right) \leq C_{A}(H)$. It follows that $C_{A}(H)$ is a subspace of $A$, as a vector space over $\operatorname{End}_{G} A$, and we put $\varphi_{G, H}(A):=\operatorname{dim}_{\operatorname{End}_{G} A} C_{A}(H)$. If $A$ is non-trivial, $C_{A}(H)<A$ as $H^{G}=G$. Hence $\varphi_{G}(A)-\varphi_{G, H}(A) \neq 0$ and, for such an $A$, we define

$$
h_{G, H}(A):=\left[\frac{\delta_{G}(A)-1+\varphi_{G}(A)}{\varphi_{G}(A)-\varphi_{G, H}(A)}\right]+1 .
$$

In order to compute $\tilde{\eta}_{\mathfrak{⿰}}(G)$, we let

$$
\tilde{\Omega}_{\widetilde{\wp}}(G):=\{A \in \Omega(G) \mid A \text { is } \widetilde{\wp} \text {-eccentric }\},
$$

and note that any $A \in \tilde{\Omega}_{\mathfrak{\wp}}(G)$ is non-trivial. We let $H$ be an $\widetilde{\wp}$-normalizer and show that

$$
\tilde{\eta}_{\mathfrak{\jmath}}(G)=\max \left\{\max _{A \in \tilde{\Omega}_{\widetilde{\gamma}}(G)}\left\{h_{G, H}(A)\right\}, 1\right\} .
$$

Since a saturated formation $\mathfrak{\wp}$ is a Schunck class and an $\mathfrak{\wp}$-projector contains an $\mathfrak{\wp}$ normalizer, $\eta_{\mathfrak{F}}(G) \leq \tilde{\eta}_{\mathfrak{\wp}}(G)$. Our formulas give $\eta_{\mathfrak{X}}(G) \leq d(G)$. The functions $d, \eta_{\mathfrak{X}}, \tilde{\eta}_{\mathfrak{\wp}}$ and the gaps in the above inequalities have no upper bounds. For example let $G$ be the semidirect product $\left(C_{2} \times C_{2}\right)^{n} \operatorname{Sym}(3)$, where Sym (3) acts on each direct factor in the natural way. In the final section of the paper, we show that, if $\mathfrak{U}$ is the formation of supersoluble groups,

$$
\eta_{\mathfrak{H}}(G)=d(G)=\tilde{\eta}_{\mathfrak{I}}(G)=\left[\frac{n-1}{2}\right]+2
$$

on the other hand, if $\mathfrak{N}$ is the formation of nilpotent groups,

$$
\eta_{\Re}(G)=2, \quad d(G)=\left[\frac{n-1}{2}\right]+2, \quad \tilde{\eta}_{\mathfrak{N}}(G)=n+2 .
$$

2. Preliminary results. We shall make repeated use of the fact that a minimal normal subgroup $N$ of $G$ is abelian. It follows immediately that, if $N$ has a supplement $L \neq G$, then $L$ is a complement of $N$ and $L$ is a maximal subgroup of $G$. 
Lemma 2.1. Let $N$ be a minimal normal subgroup of $G$ and let $\left\langle H_{1}, \ldots, H_{r}\right\rangle$ be a complement of $N$, where each $H_{i}$ is a subgroup. Then the set

$$
M:=\left\{\left(n_{1}, \ldots, n_{r}\right) \in N^{r} \mid\left\langle H_{1}^{n_{1}}, \ldots, H_{r}^{n_{r}}\right\rangle \text { is a complement of } N\right\}
$$

is a union of cosets of $C_{N}\left(H_{1}\right) \times \ldots \times C_{N}\left(H_{r}\right)$. Moreover, for $\left(m_{1}, \ldots, m_{r}\right)$, $\left(m_{1}^{\prime}, \ldots, m_{r}^{\prime}\right) \in M$ we have

$$
\left\langle H_{1}^{m_{1}}, \ldots, H_{r}^{m_{r}}\right\rangle=\left\langle H_{1}^{m_{1}^{\prime}}, \ldots, H_{r}^{m_{r}^{\prime}}\right\rangle \Longleftrightarrow m_{i} \equiv m_{i}^{\prime} \bmod C_{N}\left(H_{i}\right), \text { for each } i=1, \ldots, r .
$$

Proof. We note that $\left[N_{N}\left(H_{i}\right), H_{i}\right] \leq N \cap H_{i}=\{1\}$ forces $N_{N}\left(H_{i}\right)=C_{N}\left(H_{i}\right)$, for each $i$. Now let $\left(n_{1}, \ldots, n_{r}\right) \in N^{r}$ be such that $\left\langle H_{1}, \ldots, H_{r}\right\rangle=\left\langle H_{1}^{n_{1}}, \ldots, H_{r}^{n_{r}}\right\rangle$ and assume $H_{i} \neq H_{i}^{n_{i}}$, for some $i$. It follows that $H_{i}<\left\langle H_{i}, H_{i}^{n_{i}}\right\rangle \leq H_{i} N$, $\left\langle H_{i}, H_{i}^{n_{i}}\right\rangle \cap N \neq\{1\}$, a contradiction. We conclude that $n_{i} \in N_{N}\left(H_{i}\right)=C_{N}\left(H_{i}\right)$, for each $i$.

In the following $H$ denotes a subgroup of $G$ such that $H^{G}=G$ and, for each homomorphism $\epsilon, \eta(\epsilon(H), \epsilon(G))$ denotes the minimal number of conjugates of $\epsilon(H)$ that generate $\epsilon(G)$. We recall that, for a complemented minimal normal subgroup $N$ of $G,|\operatorname{Der}(G / N, N)|$ coincides with the number of complements of $N$ in $G$.

Lemma 2.2. Let $N$ be a minimal normal subgroup of $G=H^{G}$ and let $r:=\eta(N H / N, G / N)$. We have

(i) $r \leq \eta(H, G) \leq r+1$;

(ii) if $\eta(H, G)=r+1, H$ is contained in a complement of $N$ and

$$
\left|N / C_{N}(H)\right|^{r} \leq|\operatorname{Der}(G / N, N)|
$$

(iii) if $N$ is complemented and every complement of $N$ contains a conjugate of $H$,

$$
\left|N / C_{N}(H)\right|^{r} \geq|\operatorname{Der}(G / N, N)|
$$

and

$$
\eta(H, G)=r+1 \Longleftrightarrow\left|N / C_{N}(H)\right|^{r}=|\operatorname{Der}(G / N, N)|
$$

Proof. (i) Clearly $r \leq \eta(H, G)$. Let $\left(1, g_{2}, \ldots, g_{r}\right) \in G^{r}$ be such that $G=\left\langle N, H, H^{g_{2}}, \ldots, H^{g_{r}}\right\rangle$, and assume that $r<\eta(H, G)$. Then $L:=\left\langle H, H^{g_{2}}, \ldots, H^{g_{r}}\right\rangle$ is a complement of $N$. In particular $N$ does not normalize $H$, for otherwise $N$ would normalize $L$, contrary to the assumption that $G$ is generated by the conjugates of $H$. Hence there exists $n \in \mathbb{N}$ such that $H<\left\langle H, H^{n}\right\rangle \leq H N$. It follows that $\left\langle H, H^{n}\right\rangle \cap N \neq\{1\}$ and $G=\left\langle H, H^{n}, H^{g_{2}}, \ldots, H^{g_{r}}\right\rangle$. We conclude that $\eta(H, G)=r+1$.

(ii) In the previous notation, $L$ is a complement of $N$ that contains $H$. Moreover, for each $\left(n_{1}, n_{2}, \ldots, n_{r}\right) \in N^{r},\left\langle H^{n_{1}}, H^{g_{2} n_{2}}, \ldots, H^{g_{r} n_{r}}\right\rangle$ is a supplement and hence a complement of $N$. By Lemma 2.1 the complements of this form are exactly $\left|N / C_{N}(H)\right|^{r}$. 
(iii) Let $\ell_{1}=1$ and let $L=\left\langle H^{\ell_{1}}, H^{\ell_{2}}, \ldots, H^{\ell_{r}}\right\rangle$ be a complement of $N$ that contains $H$. The first part of the statement follows from Lemma 2.1 if we show that each complement $Y$ of $N$ is of the form $Y=\left\langle H^{y_{1}}, H^{y_{2}}, \ldots, H^{y_{r}}\right\rangle$, where $y_{i} \equiv \ell_{i}(\bmod N)$, for each $i$. For this purpose, we may assume that $H \leq Y$. Denote by $\psi: G \rightarrow Y$ the projection such that $y_{i}:=\psi\left(\ell_{i}\right) \equiv \ell_{i}(\bmod N)$, for each $i$, and $\left\langle H^{y_{1}}, H^{y_{2}}, \ldots, H^{y_{r}}\right\rangle$ is a supplement of $N$ contained in $Y$. We conclude that $Y=\left\langle H^{y_{1}}, H^{y_{2}}, \ldots, H^{y_{r}}\right\rangle$. Combining this with (ii) we have that $\eta(H, G)=r+1$ forces $\left|N / C_{N}(H)\right|^{r}=|\operatorname{Der}(G / N, N)|$. Conversly let $\left|N / C_{N}(H)\right|^{r}=|\operatorname{Der}(G / N, N)|$ and assume $G=\left\langle H, H^{g_{2}}, \ldots, H^{g_{r}}\right\rangle$, for some $\left(1, g_{2}, \ldots, g_{r}\right) \in G^{r}$. If $L$ is a complement of $N$ that contains $H$ and $\lambda: G \rightarrow L$ is the projection, we have $L=\lambda(G)=\left\langle H, H^{\lambda\left(g_{2}\right)}, \ldots, H^{\lambda\left(g_{r}\right)}\right\rangle$. By what has been proved above, each complement of $N$ is of the form $\left\langle H^{n_{1}}, H^{\lambda\left(g_{2}\right) n_{2}}, \ldots, H^{\lambda\left(g_{r}\right) n_{r}}\right\rangle$, for some $\left(n_{1}, n_{2}, \ldots, n_{r}\right) \in N^{r}$. On the other hand, by Lemma 2.1 and our hypothesis, the subgroup $\left\langle H^{n_{1}}, H^{\lambda\left(g_{2}\right) n_{2}}, \ldots, H^{\lambda\left(g_{r}\right) n_{r}}\right\rangle$ must be a complement of $N$, for each $\left(n_{1}, n_{2}, \ldots, n_{r}\right) \in N^{r}$. From $g_{i} \equiv \lambda\left(g_{i}\right)(\bmod N)$, it follows that $G=\left\langle H, H^{g_{2}}, \ldots, H^{g_{r}}\right\rangle$ is a complement of $N$, a contradiction.

As above we let

$$
\Omega(G):=\{\text { non-isomorphic, irreducible, complemented } G \text {-modules }\}
$$

and, for each non-trivial $G$-module $A \in \Omega(G)$, we let

$$
h_{G, H}(A):=\left[\frac{\delta_{G}(A)-1+\varphi_{G}(A)}{\varphi_{G}(A)-\varphi_{G, H}(A)}\right]+1 .
$$

Moreover we say that a complemented chief factor $M_{1} / N_{1}$ of $G$ avoids $H$ when $M_{1} \cap H \leq N_{1}$.

Theorem 2.3. Let $G=H^{G}$ and assume that $H$ satisfies the following conditions:

(i) if $M_{1} / N_{1}$ is a complemented chief factor of $G$ that avoids $H$, then every complement of $M_{1} / N_{1}$ in $G / N_{1}$ contains a conjugate of $N_{1} H / N_{1}$;

(ii) if $M_{1} / N_{1}$ and $M_{2} / N_{2}$ are $G$-isomorphic complemented chief factors of $G$, then $M_{1} / N_{1}$ avoids $H$ if and only if $M_{2} / N_{2}$ avoids $H$.

Then the set $\Omega_{H}(G):=\{A \in \Omega(G) \mid H$ avoids $A\}$ is well defined and

$$
\eta(H, G)=\max \left\{\max _{A \in \Omega_{H}(G)}\left\{h_{G, H}(A)\right\}, 1\right\} .
$$

Proof. We note that $\Omega_{H}(G)$ is well defined in virtue of (ii). The result is clear if $G$ has prime order, and so we argue by induction on the order of $G$. Let $N$ be a minimal normal subgroup of $G$ and let $\bar{G}:=G / N, \bar{H}:=N H / N$. As $\bar{H}$ satisfies the hypothesis above as a subgroup of $\bar{G}$, we may assume that

$$
\eta(\bar{H}, \bar{G})=\max \left\{\max _{A \in \Omega_{\bar{H}}(\bar{G})}\left\{h_{\bar{G}, \bar{H}}(A)\right\}, 1\right\} .
$$

Each $A \in \Omega_{\bar{H}}(\bar{G})$ is, by inflation, an irreducible, complemented $G$-module which avoids $H$. Moreover, if $A_{1}$ and $A_{2}$ are distinct elements of $\Omega_{\bar{H}}(\bar{G})$, they are not 
isomorphic as $G$-modules. It follows that $\Omega_{\bar{H}}(\bar{G})$ can be considered as a subset of $\Omega_{H}(G)$. A chief series of $G$ that includes $N$ gives rise, in a natural way, to a chief series of $\bar{G}$. Considering this fact, it follows easily that for each $A \in \Omega_{\bar{H}}(\bar{G})$ that is not $G$-isomorphic to $N, \delta_{G}(A)=\delta_{\bar{G}}(A)$ and $h_{G, H}(A)=h_{\bar{G}}, \bar{H}(A)$. On the other hand, if $A$ is $G$-isomorphic to $N$, then $\delta_{G}(A)-1 \leq \delta_{\bar{G}}(A) \leq \delta_{G}(A)$.

Case 1. $N$ is not complemented or $N \cap H \neq\{1\}$.

Clearly $\Omega_{H}(G)=\Omega_{\bar{H}}(\bar{G})$ and, for each $A \in \Omega_{H}(G)$, we have $h_{G, H}(A)=h_{\bar{G}, \bar{H}}(A)$. Hence, by Lemma 2.2 (ii)

$$
\eta(H, G)=\eta(\bar{H}, \bar{G})=\max \left\{\max _{A \in \Omega_{\bar{H}}(\bar{G})}\left\{h_{\bar{G}, \bar{H}}(A)\right\}, 1\right\}=\max \left\{\max _{A \in \Omega_{H}(G)}\left\{h_{G, H}(A)\right\}, 1\right\} .
$$

Case 2. $N$ is complemented and $N \cap H=\{1\}$.

Each complement of $N$ contains a conjugate of $H$. In particular, $N$ is not central, as $H^{G}=G$. By Lemma 2.2, $\eta(H, G)=\eta(\bar{H}, \bar{G}):=r$, or $\eta(H, G)=r+1$. Also, we have

$$
|\operatorname{Der}(\bar{G}, N)| \leq\left|N / C_{N}(H)\right|^{r}=\left|\operatorname{End}_{G} N\right|^{\left(\varphi_{G}(N)-\varphi_{G, H}(N)\right) r}
$$

and equality holds if and only if $\eta(H, G)=r+1$. Now, by [2, Satz 3],

$$
|\operatorname{Der}(\bar{G}, N)|=|N|\left|\operatorname{End}_{G} N\right|^{\delta_{\bar{G}}(N)}=\left|\operatorname{End}_{G} N\right|^{\varphi_{G}(N)+\delta_{G}(N)-1} .
$$

It follows that

$$
\frac{\varphi_{G}(N)+\delta_{G}(N)-1}{\varphi_{G}(N)-\varphi_{G, H}(N)} \leq r
$$

with equality if and only if $\eta(H, G)=r+1$. Hence either $h_{G, H}(N) \leq r=\eta(H, G)$ or $h_{G, H}(N)=\eta(H, G)=r+1$. In both cases we have

$$
\eta(H, G)=\max \left\{h_{G, H}(N), r\right\} .
$$

We may assume that $\Omega_{H}(G)=\Omega_{\bar{H}}(\bar{G}) \cup\{N\}$. As $h_{G, H}(N) \geq h_{\bar{G}, \bar{H}}(N)$ and, for each $A \in \Omega_{H}(G)-\{N\}, h_{G, H}(A)=h_{\bar{G}, \bar{H}}(A)$, we obtain

$$
\begin{aligned}
\eta(H, G) & =\max \left\{h_{G, H}(N), r\right\}=\max \left\{h_{G, H}(N), \max _{A \in \Omega_{\bar{H}}(\bar{G})}\left\{h_{\bar{G}, \bar{H}}(A)\right\}, 1\right\} \\
& =\max \left\{h_{G, H}(N), \max _{A \in \Omega_{\bar{H}}(\bar{G})-\{N\}}\left\{h_{\bar{G}, \bar{H}}(A)\right\}, 1\right\}=\max \left\{\max _{A \in \Omega_{H}(G)}\left\{h_{G, H}(A)\right\}, 1\right\} .
\end{aligned}
$$

3. The function $\eta_{\mathfrak{X}}$. Let $\subseteq$ be the universe of finite soluble groups. A class $\mathfrak{X}$ in $\subseteq$ is said to be a Schunck class if it consists precisely of those groups whose primitive epimorphic images are in $\mathfrak{X}$. Here, by a primitive group, we mean a group $P$ with a maximal subgroup $M$ such that $\operatorname{Core}_{P}(M)=\{1\}$. A subgroup $H$ of $G$ is an $\mathfrak{X}$-pro- 
jector if $\epsilon(H)$ is $\mathfrak{X}$-maximal in $\epsilon(G)$, for any homorphism $\epsilon$. In particular $\epsilon(H)$ is an $\mathfrak{X}$-projector of $\epsilon(G)$. The $\mathfrak{X}$-projectors of $G$ form a unique conjugacy class, denoted by $\operatorname{Proj}_{\mathfrak{X}}(G)$. See $[1,3.21]$

Lemma 3.1. Let $M_{1} / N_{1}$ be a complemented chief factor of $G$. For any $H \in \operatorname{Proj}_{\mathfrak{X}}(G)$, the following conditions are equivalent:

(i) every complement of $M_{1} / N_{1}$ in $G / N_{1}$ contains a conjugate of $H N_{1} / N_{1}$;

(ii) $H$ avoids $M_{1} / N_{1}$.

Proof. We show that (ii) implies (i), the converse being obvious. Since $H N_{1} / N_{1} \in \operatorname{Proj}_{\mathfrak{X}}\left(G / N_{1}\right)$, we may replace $G$ by $G / N_{1}, H$ by $H N_{1} / N_{1}$ and assume that $N_{1}=\{1\}, M_{1}$ is a minimal normal subgroup of $G$. Let $L_{1}$ be a complement of $M_{1}$ and let $K$ be an $\mathfrak{X}$-projector of $L_{1}$. Then $H M_{1} / M_{1}$ and $K M_{1} / M_{1}$ are $\mathfrak{X}$-projectors of $G / M_{1}$, so that up to conjugation, $H M_{1}=K M_{1}$. It follows that $H$ is an $\mathfrak{X}$-projector of $K M_{1}$, by 3.22 (a) of [1]. As $M_{1}$ is nilpotent, $K M_{1} / M_{1} \simeq K$ is in $\mathfrak{X}$ and $H$ avoids $M_{1}$; from 3.23 (c) of [1] we have $\{K\}=\operatorname{Proj}_{\mathfrak{X}}(K) \subseteq \operatorname{Proj}_{\mathfrak{X}}\left(K M_{1}\right)$. Hence $K$ is an $\mathfrak{X}$ projector of $K M_{1}$. We conclude that $H$ and $K$ are conjugate in $K M_{1}$.

Lemma 3.2. Assume that $M_{1} / N_{1}$ and $M_{2} / N_{2}$ are G-isomorphic complemented chief factors of $G$. For any $H \in \operatorname{Proj}_{\mathfrak{X}}(G), H$ avoids $M_{1} / N_{1}$ if and only if it avoids $M_{2} / N_{2}$.

Proof. Let $C:=C_{G}\left(M_{1} / N_{1}\right)=C_{G}\left(M_{2} / N_{2}\right)$ and consider the following semidirect products. Relative to the conjugation action, we have

$$
E_{1}:=\left(M_{1} / N_{1}\right)(G / C) \simeq E_{2}:=\left(M_{2} / N_{2}\right)(G / C)
$$

Note that $M_{i} / N_{i}$ is the unique minimal normal subgroup of $E_{i}$ as it is selfcentralizing $(i=1,2)$. Let $L_{i} / N_{i}$ be complements of $M_{i} / N_{i}$ in $G / N_{i}$, and consider the homomorphisms

$$
\epsilon_{i}: G=M_{i} L_{i} \rightarrow E_{i} \text { such that } m_{i} \ell_{i} \mapsto\left(N_{i} m_{i}, C \ell_{i}\right), \quad(i=1,2) .
$$

Clearly

$$
\epsilon_{i}\left(M_{i}\right)=M_{i} / N_{i} \text { and } \epsilon_{i}\left(L_{i}\right)=C L_{i} / C=G / C \text { as } M_{i} \leq C .
$$

In particular $\epsilon_{1}$ and $\epsilon_{2}$ are epimorphisms. Suppose that $H \cap M_{1} \leq N_{1}$. By the previous lemma we may assume that $H \leq L_{1}$ and hence $\epsilon_{1}(H) \leq G / C$. It follows that $\epsilon_{1}(H)$ intersects trivially the unique minimal normal subgroup $M_{1} / N_{1}$ of $E_{1}$. As $\epsilon_{i}(H)$ is an $\mathfrak{X}$-projector of $E_{i}(i=1,2), \epsilon_{2}(H)$ also intersects trivially the unique minimal normal subgroup $M_{2} / N_{2}$ of $E_{2}$. On the other hand, $\epsilon_{2}\left(H \cap M_{2}\right) \leq M_{2} / N_{2}$. Hence $H \cap M_{2} \leq \operatorname{ker} \epsilon_{2}=C \cap L_{2}$. We conclude that $H \cap M_{2} \leq M_{2} \cap L_{2}=N_{2}$.

We recall that, for a class $\mathfrak{X}$, the set $\pi$ of prime numbers $p$ such that $\mathbb{Z}_{p}$ is in $\mathfrak{X}$ is called the characteristic of the class.

THEOREM 3.3. Let $\mathfrak{X}$ be a Schunck class of characteristic $\pi$ and let $G$ be a $\pi$-group. 
(i) $G$ is generated by the $\mathfrak{X}$-projectors;

(ii) For $H \in \operatorname{Proj}_{\mathfrak{X}}(G)$, the set $\Omega_{\mathfrak{X}}(G):=\{A \in \Omega(G) \mid H$ avoids $A\}$ is well defined. Also

$$
\eta_{\mathfrak{X}}(G)=\max \left\{\max _{A \in \Omega_{\mathfrak{X}}(G)}\left\{h_{G}(A)\right\}, 1\right\} .
$$

Moreover, for each $A \in \Omega_{\mathfrak{X}}(G), \theta_{G}(A)=0$. Hence $h_{G}(A)=\left[\frac{\delta_{G}(A)-1}{\varphi_{G}(A)}\right]+2$.

Proof. The image of $H$ in $G / H^{G}$ is the identity subgroup and it is $\mathfrak{X}$-maximal. It follows that $G / H^{G}$ is a $\pi^{\prime}$-group; i.e. $G=H^{G}$. Combining this observation with 3.1 and 3.2, we see that $H$ satisfies the hypothesis of Theorem 2.3. For $A \in \Omega_{\mathfrak{X}}(G)$, let $M_{1} / N_{1}$ be a complemented chief factor of $G$ that is $G$-isomorphic to $A$. Now $H N_{1} / N_{1} \in \operatorname{Proj}_{\mathfrak{X}}\left(G / N_{1}\right)$ is selfnormalizing in $G / N_{1}$, by 4.8 of [1]. From this fact and the condition $H \cap M_{1} \leq N_{1}$, it follows easily that $C_{M_{1} / N_{1}}(H)=\{1\}=C_{A}(H)$. Hence $\varphi_{G, H}(A)=\theta_{G}(A)=0$, for each $A \in \Omega_{\mathfrak{X}}(G)$. The result is now a special case of 2.3 .

Comparing this Theorem with the result of Gaschütz for the minimal number $d(G)$ of generators for $G$, one has immediately the following result.

COROLlary 3.4. $\eta_{\mathfrak{X}}(G) \leq d(G)$.

4. The function $\tilde{\eta}_{\mathfrak{r}}$. We need some technical definitions and results: for consistency and proofs we refer to [1]. Let $\mathfrak{F}$ be a saturated formation in $\mathfrak{S}$; i.e. a nonempty class of finite soluble groups, closed with respect to epimorphic images and subdirect products, with the following additional property: whenever $F / \Phi(F)$ is in $\mathfrak{\wp}$, then also $F$ is in $\mathfrak{F}(\Phi(F)$ being the Frattini subgroup). We assume further that $\mathfrak{\wp}$ has characteristic the set $\mathbb{P}$ of all primes. Under these assumptions, $\mathfrak{F}$ is a Schunck class and there exists a function $f: \mathbb{P} \rightarrow$ fformations $\}$ with the following properties. For each prime $p,(1) f(p) \subseteq \mathfrak{F}$ consists of those groups which have a normal $p$-subgroup with quotient in $f(p)$; (2) a group $F$ is in $\mathfrak{\Im}$ if and only if $F / C_{F}(L / K) \in f(p)$, for each chief factor $L / K$ of $F$ such that $p|| L / K \mid$.

A chief factor $M_{1} / N_{1}$ of $G$ is called $\Im$-central if and only if $p|| M_{1} / N_{1} \mid \Rightarrow G / C_{G}\left(M_{1} / N_{1}\right) \in f(p)$. If this is not the case, then $M_{1} / N_{1}$ is $\mathfrak{\wp}$-eccentric. Since $\{1\}$ is in $f(p)$, for each $p$, any central chief factor is $\widetilde{\lessgtr}$-central.

Let $\Sigma$ be a Hall system of $G$ and, for each prime $p$ dividing the order of $G$, denote by $G_{p^{\prime}}$ the Hall $p^{\prime}$-subgroup of $G$ in $\Sigma$. An $\widetilde{\lessgtr}$-normalizer $H$ of $G$ can thus be defined by

$$
H:=\bigcap_{p \| G \mid} N_{G}\left(G_{p^{\prime}} \cap G^{f(p)}\right)
$$

where $G^{f(p)}$ denotes the unique normal subgroup of $G$ minimal with respect to $G / G^{f(p)}$ in $f(p)$. The $\mathfrak{\wp}$-normalizers of $G$ form a unique conjugacy class. Moreover, if $H$ is an $\mathfrak{\wp}$-normalizer of $G$, then $\epsilon(H)$ is an $\mathfrak{\wp}$-normalizer of $\epsilon(G)$, for each homomorphism $\epsilon$.

LeMma 4.1. Let $H$ be an $\mathfrak{\wp}$-normalizer of $G$ and let $M_{1} / N_{1}$ be a complemented chief factor of $G$. Then the following conditions are equivalent: 
(i) $M_{1} / N_{1}$ is $\mathfrak{\Im}$-eccentric;

(ii) $H$ avoids $M_{1} / N_{1}$;

(iii) every complement of $M_{1} / N_{1}$ in $G / N_{1}$ contains a conjugate of $H N_{1} / N_{1}$.

Proof. (i) $\Longleftrightarrow$ (ii). See [1, p. 401].

(ii) $\Rightarrow$ (iii). As $H N_{1} / N_{1}$ is an $\widetilde{\wp}$-normalizer of $G / N_{1}$, as usual we may assume that $N_{1}=\{1\}$ and $M_{1}$ is a minimal normal subgroup of $G$. We need a definition. A maximal subgroup $K$ of a group $F$ is called $\mathfrak{\wp}$-critical if $F / K_{F} \notin \mathfrak{\wp}$ and $F=K \operatorname{Fit}(F)$. Now let $L$ be a complement of $M_{1}$ and let $T$ be an $\mathfrak{\wp}$-normalizer of $L$. Then $T \in \mathfrak{\wp}$ and there exists a chain

$$
T=L_{r}<\ldots<L_{1}=L
$$

where each $L_{i}$ is maximal in $L_{i-1}$ and $\mathfrak{\wp}$-critical by [1, 3.8]. Since the $p$-group $M_{1}$ is $\Im$-eccentric, $G / C_{G}\left(M_{1}\right) \notin f(p)$. Now $L_{G} \leq C_{G}\left(M_{1}\right)$ gives

$$
\frac{G / L_{G}}{C_{G / L_{G}}\left(L_{G} M_{1} / L_{G}\right)} \simeq \frac{G}{C_{G}\left(M_{1}\right)} \notin f(p) .
$$

As $L_{G} M_{1} / L_{G} \simeq M_{1}$ is a minimal normal subgroup of $G / L_{G}$, it follows that

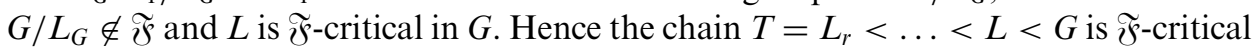
and, again by [1], $T$ is an $\mathfrak{\wp}$-normalizer in $G$. We conclude that $T$ is conjugate to $H$.

(iii) $\Rightarrow$ (ii). This is clear.

THEOREM 4.2. Let $\mathfrak{F}$ be a saturated formation of characteristic $\mathbb{P}$.

(i) $G$ is generated by the $\mathfrak{\wp}$-normalizers;

(ii) $\tilde{\eta}_{\mathfrak{\wp}}(G)=\max \left\{\max _{A \in \tilde{\Omega}(G)}\left\{h_{G, H}(A)\right\}, 1\right\}$,

where $\tilde{\Omega}_{\mathfrak{\wp}}(G):=\{A \in \Omega(G) \mid A$ is $\mathfrak{\wp}$-eccentric $\}$ and $H$ is an $\mathfrak{\wp}$-normalizer.

Proof. Since $\widetilde{F}$ has characteristic $\mathbb{P}, H^{G}=G$ by [1, p. 401]. By the previous Lemma we can apply Theorem 2.3 , with $\Omega_{H}(G)=\tilde{\Omega}_{\widetilde{\gamma}}(G)$.

In the case of a saturated formation, the set $\Omega_{\mathfrak{F}}(G)$ of Theorem 3.3 is better characterized in the following way.

Lemma 4.3. Let $H$ be an $\mathfrak{\wp}$-projector of $G$. Then

$$
\Omega_{\mathfrak{\wp}}(G)=\left\{A \in \Omega_{H}(G) \mid \text { every } H \text {-chieffactor of } A \text { is } \mathfrak{\wp} \text {-eccentric in } A H\right\} \text {. }
$$

Proof. Let $A \simeq M_{1} / N_{1}$, a complemented chief factor of $G$. The $H$-chief factors of $A$ coincide with the $H M_{1} / N_{1}$-chief factors of the normal subgroup $M_{1} / N_{1}$ of $G / N_{1}$. Now $H N_{1} / N_{1}$ is an $\mathfrak{\wp}$-projector of $G / N_{1}$ and hence of $H M_{1} / N_{1}$. As $M_{1} / N_{1}$ is a normal nilpotent subgroup of $H M_{1} / N_{1}$, with quotient $H /\left(H \cap M_{1}\right)$ in $\mathfrak{F}$, it follows that $H N_{1} / N_{1}$ is an $\mathfrak{\wp}$-normalizer of $H M_{1} / N_{1}$. (See $[\mathbf{1}, 4.2]$.) Hence $H N_{1} / N_{1}$ covers the $\mathfrak{\wp}$-central chief factors of $H M_{1} / N_{1}$ and avoids the $\mathfrak{\wp}$-eccentric ones. By definition

$$
A \in \Omega_{\widetilde{\gamma}}(G) \Longleftrightarrow M_{1} \cap H \leq N_{1} \Longleftrightarrow\left|\frac{H N_{1}}{N_{1}} \cap \frac{M_{1}}{N_{1}}\right|=1 .
$$


We conclude that $A \in \Omega_{\widetilde{\wp}}(G)$ if and only if all the $H$-chief factors of $A$ are $\mathfrak{\wp}$ eccentric.

5. Examples. Denote by $\mathfrak{N}$ the saturated formation of nilpotent groups. $\mathfrak{N}$ is local with respect to the formation function $f$ such that $f(p)=\{1\}$, for each prime $p$. It follows that a chief factor is central if and only if it is $\mathfrak{i}$-central. The $\mathfrak{N}$-projectors are the Carter subgroups and the $\mathfrak{i}$-normalizers are the system normalizers. If $A=M_{1} / N_{1}$ is a chief factor and $H$ is a Carter subgroup of $G$, then $H$ avoids $M_{1} / N_{1} \Longleftrightarrow C_{A}(H)=\{1\}$. As a matter of fact, $H \cap M_{1} \leq N_{1}$ implies that $C_{A}(H)=\{1\}$, since $H N_{1} / N_{1}$ is selfnormalizing in $G / N_{1}$. On the other hand,

$$
H \cap M_{1} \not \leq N_{1} \Rightarrow\{1\}<Z\left(\frac{H N_{1}}{N_{1}}\right) \cap \frac{M_{1}}{N_{1}} \leq C_{A}(H) .
$$

Hence, in this case, we have

$$
\Omega_{\Re}(G)=\left\{A \in \Omega(G) \mid C_{A}(H)=\{1\}\right\},
$$

where $H$ is a Carter subgroup of $G$, and

$$
\tilde{\Omega}_{\Re}(G)=\{A \in \Omega(G) \mid A \text { non trivial } G \text {-module }\} .
$$

REMARK. Let $\mathfrak{\lessgtr}$ be a saturated formation of characteristic $\pi, G$ a $\pi$-group and $H$ an $\mathfrak{\wp}$-projector of $G$. Then $N_{G}(H)=H$ so that, for each minimal normal subgroup $N$ of $G$, we have

$$
H \cap N=\{1\} \Rightarrow C_{N}(H)=\{1\} .
$$

However, the converse is not true in general. For example, if $\mathfrak{U}$ is the formation of supersoluble groups, $G$ is the symmetric group Sym(3) and $N$ is the alternating group Alt (3), then

$$
H=G, C_{N}(H)=\{1\}, H \cap N=N
$$

Denote by $\mathfrak{U}$ the saturated formation of supersoluble groups. $\mathfrak{U}$ is local with respect to the formation function $f$ such that $f(p)=$ abelian groups of exponent dividing $(p-1)\}$, for each prime $p$. A chief factor is $\mathbb{U}$-eccentric if and only if it is not cyclic. By Lemma 4.3

$$
\Omega_{\mathfrak{l}}(G)=\{A \in \Omega(G) \mid A \text { has no cyclic } H \text {-chief factor }\},
$$

where $H$ is a $\mathbb{U}$-projector. On the other hand we have

$$
\tilde{\Omega}_{\Re}(G)=\{A \in \Omega(G) \mid A \text { non cyclic }\} .
$$

1. Let $G$ be the symmetric group Sym (4). Consider the chief series

$$
N_{4}=\{1\}<N_{3}=C_{2} \times C_{2}<N_{2}=\operatorname{Alt}(4)<N_{1}=\operatorname{Sym}(4),
$$


and let $A_{i}$ be a $G$-module $G$-isomorphic to the chief factor $N_{i} / N_{i+1}, 1 \leq i \leq 3$. The Carter subgroups of $G$ are the Sylow 2-subgroups and the system normalizers are the subgroups generated by a 2-cycle. It is easy to see that

$$
\Omega(G)=\left\{A_{1}, A_{2}, A_{3}\right\}, \quad \Omega_{\Re}(G)=\left\{A_{2}\right\}, \quad \tilde{\Omega}_{\mathfrak{N}}(G)=\left\{A_{2}, A_{3}\right\},
$$

with $h_{G}\left(A_{1}\right)=1, h_{G}\left(A_{2}\right)=h_{G}\left(A_{3}\right)=2$ and $h_{G, H}\left(A_{2}\right)=2, h_{G, H}\left(A_{3}\right)=3$, where $H$ is a system normalizer. It follows that

$$
d(G)=\eta_{\Re}(G)=2, \quad \tilde{\eta}_{\Re}(G)=3 .
$$

2. Let $G$ be the semidirect product $\left(C_{2} \times C_{2}\right)^{n} \operatorname{Sym}(3)$, where Sym (3) acts on each direct factor in the natural way. In this case $\Omega(G)=\left\{A_{1}, A_{2}, A_{3}\right\}$, where

$$
\begin{gathered}
A_{1} \text { is } G \text {-isomorphic to }\left(\left(C_{2} \times C_{2}\right)^{n} \operatorname{Sym}(3)\right) /\left(\left(C_{2} \times C_{2}\right)^{n} \operatorname{Alt}(3)\right), \\
A_{2} \text { is } G \text {-isomorphic to }\left(\left(C_{2} \times C_{2}\right)^{n} \operatorname{Alt}(3)\right) /\left(C_{2} \times C_{2}\right)^{n}
\end{gathered}
$$

and $A_{3}$ is $G$-isomorphic to $C_{2} \times C_{2}$. Since $\delta_{G}\left(A_{1}\right)=\delta_{G}\left(A_{2}\right)=1$ and $\delta_{G}\left(A_{3}\right)=n$ we have

$$
h_{G}\left(A_{1}\right)=1, \quad h_{G}\left(A_{2}\right)=2, \quad h_{G}\left(A_{3}\right)=\left[\frac{n-1}{2}\right]+2 .
$$

Again the Carter subgroups of $G$ are the Sylow 2-subgroups while the subgroup $H_{1}$ generated by a 2-cycle of Sym (3) is a system normalizer. In this case we have

$$
\Omega_{\mathfrak{N}}(G)=\left\{A_{2}\right\}, \quad \tilde{\Omega}_{\mathfrak{N}}(G)=\left\{A_{2}, A_{3}\right\}
$$

and $h_{G, H_{1}}\left(A_{2}\right)=2, h_{G, H_{1}}\left(A_{3}\right)=n+2$. It follows that

$$
\eta_{\mathfrak{N}}(G)=2, \quad d(G)=\left[\frac{n-1}{2}\right], \quad \tilde{\eta}_{\mathfrak{M}}(G)=n+2 .
$$

On the other hand the $\mathfrak{U}$-projectors and the $\mathfrak{H}$-normalizers coincide and are precisely the complements in $G$ of the normal subgroup $\left(C_{2} \times C_{2}\right)^{n}$. We have

$$
\Omega_{\mathfrak{l}}(G)=\tilde{\Omega}_{\mathfrak{l}}(G)=\left\{A_{3}\right\}
$$

and $h_{G}\left(A_{3}\right)=h_{G, H_{2}}\left(A_{3}\right)=\left[\frac{n-1}{2}\right]+2$, where $H_{2}$ is a $\mathfrak{U}$-normalizer. It follows that

$$
\eta_{\mathfrak{l}}(G)=d(G)=\tilde{\eta}_{\mathfrak{l}}(G)=\left[\frac{n-1}{2}\right]+2
$$

\section{REFERENCES}

1. K. Doerk and T. Hawkes, Finite soluble groups (De Gruyter, 1992).

2. W. Gaschütz, Die Eulersche Funktion endlicher auflosbarer Gruppen, Illinois J. Math. 3 (1959), 469-476. 